\title{
Heart Failure Research and Polypharmacy
}

\author{
John Somberga, b
}

For many years the therapy for heart failure with a low ejection fraction was digoxin and diuretics with a very grim prognosis. Starting the 1980s we have been fortunate to see the introduction of evidence-based therapies that improve how patients feel, as well as reducing mortality. First there was the introduction of beta blockers showing a mortality reduction followed by angiotensin-converting enzyme (ACE) inhibitors and subsequently the angiotensin receptor blockers (ARBs). Mineralocorticoids, spironolactone and then eplerenone were found to reduce mortality though the problem of hyperkalemia is a serious impediment to therapy. Of late, the sodium glucose transporter 2 (SGLT2) inhibitor, empagliflozin, and dapagliflozin have been noted to reduce heart failure mortality.

This is quite an array of therapies for heart failure that possess challenges for clinicians to effectively introduce into patient care with tolerability and compliance issues. Further complicating heart failure therapeutics is the report of the PARADIGM - HF trial with the agent sacubitril valsartan, a fixed combination of an ARB with a neprilysin inhibitor sacubitril. The PARADIGM - HF trial was stopped prematurely after an interim analysis revealing a significant reduction in death and hospitalizations with sacubitril valsartan compared to enalapril (200 mg vs.10 mg, twice a day (bid)). Hypotension can be problematic with the sacubitril valsartan. The reduction in cardiovascular (CV) death (13\% vs. $16 \%)$ and all-cause mortality (17\% vs. $19.8 \%$ ) was very significant with the drug's approval based on a single study. While it has been said repeatedly that one study at a $\mathrm{P}<0.001$ is the same as two studies at $\mathrm{P}<0.025$, this is not the case. Each study has a unique set of factors that for unknown reasons may limit the studies generalizability to the population as a whole, and that is why for major new therapies, a second study should be undertaken to re-confirm the findings of the first. Needless to say that sacubitril valsartan is an effective therapy, although we do not know if more effective than a long-acting ACE (lisinopril or verapamil) or a long-acting ARB at a maximal dose. Additionally, in the PARADIGM - HF trial only $29 \%$ were on digoxin and $54 \%$ on a mineralocorticoid, suggesting that not all patients will be on or need to be on mineralocorticoids to see the angiotensin receptor-neprilysin inhibitor (ARNI) effect.

Manuscript submitted January 17, 2022, accepted January 19, 2022

Published online January 29, 2022

${ }^{a}$ Cardiology \& Pharmacology, Rush University, Chicago, IL 60612, USA.

Email: John_Somberg@rush.edu

${ }^{b}$ Editor-in-Chief, Cardiology Research

doi: https://doi.org/10.14740/cr1356
Further complicating the heart failure therapeutic field is the novel drug vericiguat, an oral soluble guanylate cyclase stimulator. This agent is now available in the USA for patients with reduced ejection fraction heart failure; and the drug showed a reduction in hospitalization, but did not reduce cardiovascular or total mortality (VICTORIA trial). Another agent under consideration for approval is omecamtiv mecarbil, a select cardiac myosin activator. In the GALACTIC - HF trial the drug reduced heart failure hospitalization, but did not reduce mortality.

This array of therapeutics for heart failure is a real benefit to heart failure patients, but it also poses significant issues. Should all these agents be employed simultaneously, in what sequence should they be introduced, how fast should they be titrated when dose titration is available and what agent is more effective than another within a specific class of drugs? The system we have to study drugs depends on drug developers for the most part. Pharmaceutical companies are interested in obtaining approval, not establishing optimum therapeutic approaches. What is needed is support for comparative effective studies comparing agents and different approaches to established therapies and to one another. Even the simple question of how aggressive should one be with diuretic therapy when patients are on the "four pillars" of guideline-directed therapy: ARNI, beta blockers, mineralocorticoids and SGLT2 inhibitor is unanswered. One needs to ask if the mortality benefits are truly additive among the beta blockers, sacubitril valsartan, mineralocorticoids and the SGLT2 blockers necessitating the considerable physician efforts to employ this polypharmacy and maintain patient compliance. The national comparative effectiveness research program, a component of the Affordable Care Act, would be one potential funding source for the studies that are sorrowfully needed.

\section{Financial Disclosure}

None to declare.

\section{Conflict of Interest}

None to declare.

\section{Data Availability}

The author declares that data supporting the findings of this study are available within the article. 